\title{
Zur Legitimität von Staatlichkeit : eine kosmopolitische Kritik offener Grenzen
}

In: Nida-Rümelin, Julian / Daniels, Detlef von / Wloka, Nicole (Hrsg.): Internationale Gerechtigkeit und institutionelle Verantwortung. - ISBN: 978-3-11-061586-9. - Berlin: De Gruyter, 2019.

S. 239-255

(Forschungsberichte / Interdisziplinäre Arbeitsgruppen, Berlin-Brandenburgische Akademie der Wissenschaften ; 41) 


\section{Zur Legitimität von Staatlichkeit: Eine kosmopolitische Kritik offener Grenzen}

\section{Einführung}

Grenzen sind ein konstitutives Merkmal von Staatlichkeit. Politische Gestaltungskraft gerät daher in Konflikt mit einer Praxis offener Grenzen. Dies gilt für die Globalisierungsdynamik im Zeichen entfesselter globaler Finanz- und Warenmärkte ebenso wie für den globalen Arbeitsmarkt. Eine ungebremste Mobilität von Kapital, Waren und Menschen ließe Staatlichkeit erodieren und degradierte die Politik zum bloßen Standortfaktor. Im Folgenden argumentiere ich für die Legitimität von (Staats-)Grenzen und die politische Kontrolle von Migrationsbewegungen, allerdings nicht aus einer kommunitaristischen oder gar nationalistischen, sondern aus einer kosmopolitischen Perspektive. Der politische Kosmopolitismus unterscheidet sich von einem soziologischen, ökonomischen oder kulturellen vor allem hinsichtlich der Rolle der Politik. Während andere Kosmopolitismus-Varianten (vgl. Nida-Rümelin 2006) die Globalisierung als Schwächung von Staatlichkeit generell verstehen, setzt der politische Kosmopolitismus auf die Etablierung einer globalen institutionellen Ordnung, die demokratisch legitimierte politische Gestaltungskraft auch jenseits der Nationalstaaten zulässt. Die Frage stellt sich dann, welche institutionelle Steuerung der Migration im kosmopolitischen Rahmen legitim ist. Der folgende Text stellt politiktheoretische (Abschnitt 2) und philosophisch-ethische (Abschnitt 3) Vorüberlegungen zu dieser Thematik zur Diskussion.

\section{Politische Aspekte}

Die meisten Philosophen - zumindest diejenigen, die zu dieser Frage Stellung beziehen - sind für offene Grenzen. Einige argumentieren entweder aus kom-

\footnotetext{
Dieser Artikel entspricht inhaltlich meinem Vortrag „On the Ethical Legitimacy of (State-)Borders“, gehalten beim Workshop „Justice, State, Migration“ an der Berlin-Brandenburgischen Akademie der Wissenschaften am 19. Dezember 2016. Er folgte auf den Vortrag von David Miller „Immigrants, Refugees, and the Liberal State“, der unter dem Titel „Migration, Flucht und der liberale Staat" ebenfalls in diesem Band enthalten ist. Christine Bratu danke ich für die Übersetzung des ersten Teils („Politische Aspekte“) aus dem Englischen.
}

Ә OpenAccess. (C) 2019 Julian Nida-Rümelin, publiziert von De Gruyter. (cc) BY-NC-SA Dieses Werk ist lizenziert unter der Creative Commons Attribution-NonCommercial-ShareAlike 4.0 Lizenz. 
munitaristischen oder aus nationalistischen Gründen für geschlossene Grenzen (wobei einige Vertreter einer nationalistischen Position Republikaner sind, andere Kommunitaristen). Kosmopoliten sind meistens für offene Grenzen, Anti-Kosmopoliten dagegen. Ich halte mich selbst für einen kosmopolitischen Philosophen, dennoch bin ich gegen offene Grenzen. Ich bin der Überzeugung, dass eine sinnvolle Fassung des Kosmopolitismus gegen offene Grenzen argumentieren und stattdessen die Legitimität staatlicher Grenzen akzeptieren muss. Im Folgenden werde ich versuchen, dies zu zeigen.

Kosmopoliten betrachten die Welt aus einer globalen Perspektive. Ihre Analyse beginnt mit der Frage „Was wäre eine legitime und gerechte Weltordnung?“. Ausgehend von dieser Frage diskutieren sie dann Implikationen für die Migrationspolitik. Die Prämissen für die Diskussion sind je nach Standpunkt verschieden: Nationalisten betrachten die Welt aus der Perspektive nationaler Interessen, Kommunitaristen hingegen sehen die Werte und Normen der Gemeinschaft, der sie angehören, als unhintergehbaren Ausgangspunkt. Republikaner nehmen schließlich einen dezidiert politischen Standpunkt ein, da sie annehmen, dass sich normative Rechtfertigung nur im Rahmen politischer Partizipation ergibt. Dementsprechend beschreiben sie die institutionellen Elemente, die für Partizipation konstitutiv sind. Einige Republikaner (aber nicht alle) sind in einem noch zu klärenden Sinne nationalistisch, ebenso wie einige (aber nicht alle) Kommunitaristen nationalistisch sind. Republikaner sind dagegen niemals kommunitaristisch, da sie im demos und nicht im ethnos die Quelle der Legitimität des Staates sehen.

Der Kosmopolitismus dreht sich um Fragen der globalen politischen Ordnung. ${ }^{1}$ Er diskutiert Kriterien einer gerechten Weltordnung und besteht auf dem Primat des Politischen. Zudem betrachtet er die Menschheit und ihr Interesse als Ganze und rückt dadurch die Interessen von Staaten und Gemeinschaften in den Hintergrund. Kosmopoliten sind Universalisten. Sie behaupten, dass die grundlegenden ethischen Regeln für alle Erdenbewohner gelten, unabhängig von ihrer Staatsbürgerschaft, ihrer Hautfarbe, ihrer Muttersprache oder anderen partikularen Zugehörigkeiten.

Einige Kosmopoliten argumentieren gegen den Nationalstaat. Sie behaupten, dass eine gerechte Weltordnung, ausgehend von allgemeinen ethischen Prinzipien, Nationalstaaten insgesamt abschaffen würde. Wenn man den Nationalstaat als die politische Institution definiert, die die Nation ernst nimmt - d.h. ein Volk, das ein Zugehörigkeitsgefühl verbindet, das historisch gewachsen ist, Aus-

1 Zugegebenermaßen gibt es verschiedene Formen des Kosmopolitismus, unter anderem soziologische, wie sie Beck (2000) entwickelt hat. 
gleichhandlungen rechtfertigt und nach staatlicher Verfasstheit strebt (vgl. Miller 2000) -, so sind wahrscheinlich die meisten der existierenden Staaten in der Welt keine Nationalstaaten: Denn entweder leben jene, die sich dem Staatsvolk durch ein Zugehörigkeitsgefühl verbunden fühlen, zum Teil nicht im Staat oder sind Bürger anderer Staaten, oder aber die Bürgerschaft umfasst mehr als eine Nation im oben dargestellten Sinne.

Die im 19. Jahrhundert aufkommende Idee, dass Staaten Nationalstaaten sein sollten, hat zu vielen Konflikten geführt. Einige davon eskalierten zu Kriegen und Bürgerkriegen, die sich bis in die Gegenwart ziehen. Diese Konflikte gingen zum Teil darauf zurück, dass Staatsgrenzen traditionellerweise nationalen Strukturen keine Rechnung getragen haben oder dass das Zugehörigkeitsgefühl ambivalent war oder sich gewandelt hat. Der Nationalstaat ist ursprünglich ein kommunitaristisches Konzept und basiert auf der Idee, dass es außerpolitische Entitäten gibt, die versuchen, sich als Staaten zu organisieren.

Republikaner sind keine Kommunitaristen. Ihrer Ansicht nach wird die Republik durch das Erleben gemeinsamen politischen Handelns gestiftet. Ein kommunitäres Zugehörigkeitsgefühl kann für dieses Erleben hilfreich oder hinderlich sein, aber in jedem Fall ist es hierfür nicht konstitutiv. Der Republikanismus betrachtet den demos als die Quelle der Legitimität des Staates, nicht die kulturelle Gemeinschaft, selbst wenn diese versucht, politisch zu werden.

Kommunitär ausgerichtete Nationalisten halten dagegen das ethnos für die Quelle der Legitimität des Staates. Aus pragmatischen Gründen mögen Republikaner Staaten bevorzugen, die nur eine Sprachgemeinschaft umfassen, denn für sie ist politisches Deliberieren wichtig und dies geschieht einfacher, wenn alle Beteiligten dieselbe Muttersprache sprechen. Frankreich ist hierfür ein interessantes geschichtliches Beispiel. Die Französische Revolution wurde von republikanischen und nicht von kommunitären Idealen getragen. Diese republikanischen Ideale waren eng verknüpft mit universellen, kosmopolitischen Prinzipien: Freiheit, Gleichheit, Brüderlichkeit. Dennoch stieß die Französische Revolution im Weiteren Prozesse der kulturellen Vereinheitlichung an, die in der Marginalisierung von kulturellen und sprachlichen Minderheiten resultierten.

Für einen republikanischen Kosmopoliten ist die Frage der staatlichen Organisation eine pragmatische: Wie sollten Staaten verfasst sein, damit politisches Handeln, z. B. die Rahmengebung für die Gemeinschaft durch politische Institutionen, Diskussionen und Entscheidungen, möglich und effektiv durchführbar ist? Mir scheint, dass Immanuel Kant mit seiner Annahme Recht hatte, dass ein solches Modell in globalem Maßstab nicht möglich wäre (vgl. Kant 1969). Der Weltstaat würde wahrscheinlich despotisch. Nach Kant ist der Grund hierfür, dass politische Kontrolle durch die Bürgerschaft ausgeübt werden muss; dafür muss diese aber in der Lage sein, effektiv zu intervenieren und Meinungen und Ent- 
scheidungen so zu artikulieren, dass sie für die Gemeinschaft als Ganze relevant sind.

Föderalismus im Sinne von dezentralisierten politischen Strukturen kann sowohl auf kommunitaristische als auch republikanische Überlegungen zurückgehen. Aus kommunitaristischen Gründen spricht man sich im Lichte verschiedener Sprachen sowie verschiedener kultureller und ethnischer Gruppen für föderale Strukturen aus. Aus republikanischen Gründen spricht man sich dagegen für eine staatliche Organisation aus, die politisches Handeln auf allen Ebenen effektiv ermöglicht. Hier ist es also nicht die Zugehörigkeit zu einer außerpolitischen Gemeinschaft, die für einen dezentralen föderalen Staat spricht, sondern die Komplexität politischer Entscheidungen und Handlungen. Dezentralisierung wird als ein Mittel angesehen, politische Kontrolle und politisches Handeln effektiv zu gestalten. Zwar können sich auch Republikaner dafür aussprechen, kommunitäre Strukturen ernst zu nehmen, aber nicht, weil diese Strukturen selbst Quelle politischer Legitimität wären. Vielmehr gehen Republikaner davon aus, dass die Erfahrung gemeinsamen politischen Handelns mit kommunitärem Zugehörigkeitsgefühl überlappen kann und sollte. Ein Staat, der sich selbst lediglich als ein Bündel kommunitärer Entitäten versteht, würde auf einen modus vivendi reduziert, der seine Legitimität aus den kommunitären Strukturen bezöge. Es würde unmöglich, eine gemeinsame normative Basis für politisches Handeln zu entwickeln, wenn diese Gemeinschaften zu stark in ihren Normen, Werten und Lebensformen differierten. Republikaner sprechen sich daher dafür aus, die politische Gemeinschaft statt der überlappenden kommunitären Strukturen zu stärken.

Die Weltgemeinschaft umfasst ein breites Spektrum von verschiedenen Lebensformen, Religionen, Werten und Normen. Ein globales politisches System, das auf Repräsentanten dieser Unterschiede aufbaute, würde auf den minimalen Konsens eines modus vivendi reduziert. Das ist zwar sicher besser als die gegenwärtige Situation, die in manchen Weltregionen eher dem Hobbes'schen bellum omnium contra omnes und in anderen dem langen Krieg der christlichen Konfessionen im 17. Jahrhundert ähnelt. Aber der Kosmopolitismus beschränkt sich nicht auf einen bloßen globalen modus vivendi: Kosmopoliten sollten Republikaner sein, sie sollten sich dafür aussprechen, die Möglichkeit politischen Handelns von der Ebene des Nationalstaats auf die kontinentale und globale Ebene zu heben.

Die Globalisierung hat $\mathrm{zu}$ einem sich immer weiterspinnenden weltweiten Netzwerk von ökonomischen, sozialen und kulturellen Verflechtungen geführt, das die Welt in ein globales System der Kooperation und des Konflikts verwandelt. Da der moderne Nationalstaat die Antwort auf ein sich immer weiterspinnendes Netzwerk von Kooperation und Konflikt jenseits der kommunitären Ebene war, 
sollte eine kosmopolitische politische Ordnung als Antwort auf die Entwicklungen jenseits des Nationalstaats etabliert werden. Vor zweihundert Jahren reichte die lokale Herrschaft feudaler Familien in Europa nicht mehr. Heute reicht die lokale Herrschaft der Nationalstaaten nicht mehr. Der gegenwärtige Kosmopolitismus ist dabei, politisches Handeln und politische Institutionen jenseits des Nationalstaats zu etablieren. Dem Kosmopolitismus geht es nicht darum, das gegenwärtige System der Staaten abzuschaffen, sondern darum, dieses um eine föderale Struktur zu ergänzen, die von Städten und Regionen bis hin zu Nationalstaaten reicht und die regionale Strukturen wie die Europäische Union genauso umfasst wie globale Institutionen (auch wenn Letztere noch auf wenige, schlecht organisierte Beispiele wie den Internationalen Strafgerichtshof oder den Internationalen Währungsfonds beschränkt sind). Der Kosmopolitismus zielt darauf, eine föderale Weltordnung mit institutionellen Strukturen zu erschaffen, die effektives politisches Handeln und Entscheiden auf allen Ebenen ermöglichen. Wie im Falle des einzelnen republikanischen Staates wird eine republikanische oder kosmopolitische Ordnung zur Entwicklung politischer Identitäten führen, die die bestehenden kommunitären Identitäten überlappen. Dadurch wird sie dabei helfen, eine zivile Weltgesellschaft zu etablieren.

Kommen wir nun aus einer kosmopolitischen und republikanischen Perspektive auf einige Aspekte der Ethik der Migration zu sprechen. Da es dem Kosmopolitismus nicht darum geht, einzelne Staaten abzuschaffen, sondern sie um eine föderale Weltordnung zu ergänzen, spricht sich der Kosmopolitismus für die Existenz von staatlichen Grenzen aus. Eine allgemeine Politik der offenen Grenzen nicht nur für Güter und Leistungen, sondern auch für Arbeitskräfte und Migranten im Allgemeinen wäre mit der institutionellen Struktur eines republikanischen Kosmopolitismus nicht vereinbar. Der republikanische Kosmopolitismus setzt die Möglichkeit voraus, kollektiv zu handeln, um politische Ziele zu verwirklichen. Im Allgemeinen ist ein Freihandelssystem dem politischen Handeln abträglich. Es vereinzelt das Individuum, es zerstört Strukturen der Kooperation und degradiert politische Entscheidungen zu Standortfaktoren im ökonomischen Wettbewerb. Wenn eine politische Entität versucht, im Wettbewerb mit anderen politischen Entitäten die besten Bedingungen für ökonomische Investitionen zu etablieren, verliert sie dadurch die Fähigkeit, geleitet von politischen Gründen zu handeln und gemeinsame politische Werte und Ideen zu realisieren. Politik wird zu einem bloßen Mittel ökonomischer Ziele. Wenn es für die Politik nichts mehr zu tun gibt, weil der freie Markt die Verteilung aller Güter, Leistungen, Werte, Normen und Praktiken übernimmt, werden politische Institutionen inhaltsleer. Politisches Handeln wird Teil eines großen Illusionstheaters, das den Eindruck vermittelt, es gebe politisch noch etwas zu entscheiden, wenn dies doch längst nicht mehr der Fall ist. Stattdessen sollte politisches Handeln, d.h. poli- 
tische Entscheidungen innerhalb etablierter Institutionen, dazu in der Lage sein, das Spielfeld als Ganzes zu gestalten. Ohne den Primat des Politischen gibt es keine republikanische Ordnung.

Eine Migrationspolitik, die sich für offene Grenzen ausspricht, steht in der Tradition der Freihandelsideologie und des Libertarismus. Eine solche Politik würde es politischen Institutionen unmöglich machen, eine gerechte soziale Ordnung zu erschaffen. Jede Maßnahme zum Arbeitnehmerschutz oder zur Umverteilung von Wohlstand innerhalb einer Gesellschaft würde von Migrationsbewegungen unmöglich gemacht, die durch diese Anreize ausgelöst würden. Ein weltweites Freihandelssystem der Arbeitskräfte hätte zerstörerische Auswirkungen auf entwickelte Sozialstaaten.

In dieser Hinsicht ist Migration sehr ambivalent. Für jene, die aus armen Regionen auswandern, stellt dies meist einen ökonomischen Fortschritt dar. Empirische Studien belegen aber, dass ein beträchtlicher Anteil der erfolgreichen Migranten insgesamt gesehen verliert, wenn man auch psychologische und kulturelle Aspekte einbezieht. Wichtiger noch: Die Regionen, aus denen die meisten Migranten stammen, verlieren deutlich mehr, wenn ein Großteil ihrer Bevölkerung auswandert. Globale Migration ist kein effektives Mittel im Kampf gegen globale Armut. Da ungefähr zwei Milliarden Menschen auf der Welt unter extremer Armut, d.h. unter anderem unter chronischer Unterernährung, Mangel an Trinkwasser, sozialer Exklusion, keinem oder unzureichendem Zugang zu Bildung, Arbeitslosigkeit und anderen Entbehrungen leiden, kann globale Migration in die reichen Länder das Problem der globalen Armut nicht lösen.

Die Kosten der Integration von Migranten hängen stark vom Sozialsystem des jeweiligen reichen Staates ab. ${ }^{2}$ Mit Subventionen in derselben Größenordnung ließe sich Armut weit effektiver in den Ursprungsländern bekämpfen. Migration ist in der Tat kein geeignetes Mittel, um globale Armut zu bekämpfen. In vielen Fällen leistet sie eher einen Beitrag zur globalen Armut. Wenn arme Staaten einen großen Teil ihres Haushalts in Bildung und Qualifizierungsmaßen investieren, dann aber damit konfrontiert sind, dass die Personen, die von diesen Maßnahmen profitiert haben, auswandern, führt das zum Kollaps dieser Entwicklungsstrategie. Daher sollten reiche Staaten, die von der Einwanderung hochqualifizierter

2 Die Bundesrepublik Deutschland zahlt ca. 250.000 Euro pro Migrant an die Türkei, damit diese die betreffenden Personen wieder zurücknimmt. Daher kann angenommen werden, dass die Kosten für die Integration dieser Personen in die deutsche Gesellschaft höher liegen. 
Arbeitskräfte profitieren, dazu verpflichtet werden, die Ursprungsstaaten zu kompensieren. ${ }^{3}$

Im Falle von Kriegs- und Bürgerkriegsflüchtlingen ist es Aufgabe der Weltgemeinschaft, die Anrainerstaaten, welche den Flüchtlingen Obhut gewähren, finanziell zu unterstützen. Die Integration von Kriegs- und Bürgerkriegsflüchtlingen in Länder, die Tausende von Meilen entfernt sind, macht die Situation in vielen Fällen schlimmer. Wenn der Krieg vorbei ist, werden die Flüchtlinge zurückkehren, um Häuser, Wirtschaft und die Gesellschaft als Ganze wieder aufzubauen. Wenn jene, die über finanzielle Mittel verfügen, nicht zurückkehren, weil sie mittlerweile ökonomisch und sozial in eine reiche Gesellschaft wie die USA oder einige der nördlichen und mitteleuropäischen Staaten integriert sind, wird es für die Ursprungsregionen noch schwieriger, sich vom Kriegsgeschehen zu erholen.

Reiche Länder, die die Kosten von groß angelegter Migration tragen, sollten zudem bedenken, dass die Kosten hierfür meistens von jenem Teil der Bevölkerung getragen werden, der relativ arm ist. Dieser Bevölkerungsteil leidet, weil er ökonomisch mit den Migranten auf dem Arbeitsmarkt konkurrieren muss, weil die Mieten steigen und weil er schnellen kulturellen und sozialen Wandel erlebt, den seine Mitglieder als anstrengend empfinden könnten. Reiche Bevölkerungsgruppen profitieren dagegen von Migration aus armen Ländern: Sie können Hausmädchen und Gärtner einstellen und diesen sogar noch niedrigere Löhne zahlen als unausgebildeten heimischen Arbeitskräften. Auch wenn es gute ökonomische Argumente für die Immigration gut ausgebildeter Fachkräfte in die wohlhabenden westlichen Länder gibt, sind die kulturellen und sozialen Kosten doch ungerecht verteilt.

\section{Ethische Aspekte}

Als ich in der Frühe in mein Wohnzimmer komme, um zu frühstücken, muss ich zu meinem Erstaunen feststellen, dass dort schon eine Person sitzt. Die Person ist freundlich, sympathisch, aber auch sehr bestimmt: Sie hat sich, wie sie erzählt, mit einem Dietrich Zutritt zu meiner Wohnung verschafft, sie ist obdachlos und bittet mich nun um Zustimmung, diese Wohnung in Zukunft mit mir zu teilen. Obwohl ich die schwierige Situation des Obdachlosen durchaus nachvollziehen

3 Die praktische Umsetzung dieses Vorschlags ist zweifellos kompliziert. Sinnvoll erscheint ein System, bei dem sowohl das Unternehmen, der Staat und die Migranten Ausgleichszahlungen erbringen. Gillian Brock hat hierzu einen detaillierten Vorschlag unterbreitet, vgl. Brock (2009). 
kann und er mir keineswegs unsympathisch ist, bitte ich ihn, meine Wohnung umgehend $\mathrm{zu}$ verlassen. Die meisten Leserinnen und Leser werden mir darin zustimmen, dass dies nicht nur mein juridisches, sondern auch mein moralisch begründetes Recht ist. Besteht dieses (moralische) Recht und wenn ja, aus welchen Gründen?

Um die Situation noch ein wenig zu konkretisieren: Ich bin davon überzeugt, dass der Obdachlose nicht mit dem Tod bedroht ist, wenn ich ihn aus meiner Wohnung weise. Ich bin aber zugleich davon überzeugt, dass sich die Lebenssituation des Obdachlosen deutlich verbessern würde, wenn ich seinem Begehren nachgekommen wäre. Zudem steht völlig außer Frage, dass meine Lebenssituation eine weit bessere ist als die des Obdachlosen und dass sich die Nachteile, die sich aus einer Kohabitation für mich ergäben, in Grenzen hielten, dass die Vorteile, die der Obdachlose von einer Kohabitation hätte, meine Nachteile bei weitem überwiegen würden. Denkbar wäre auch, dass ich in eine Verhandlung einträte; das Ergebnis könnte sein, dass wir uns auf einen Aufenthalt von zum Beispiel einer Woche einigten, mit Vorteilen für ihn, Nachteilen für mich, aber für beide akzeptabel. Verpflichtet wäre ich dazu allerdings offenkundig nicht.

Die legitime Grenze ist in diesem Fall durch meine Wohnung gezogen. Ich kontrolliere als Wohnungseigentümer oder Mieter den Zutritt zu dieser Wohnung und mein Status als Eigentümer oder Mieter gibt mir individuelle Rechte, darunter das Recht, den Zutritt oder den Aufenthalt zu verweigern, auch im Falle, dass die Person gute Gründe hat, sich den Zutritt oder den Aufenthalt zu wünschen, wie in diesem Fall. Verletzen die individuellen (juridischen und ethischen) Rechte des Wohnungseigentümers ein Gleichbehand1lungsprinzip? Muss nicht jede Person gleichermaßen Zutritt zu dieser Wohnung haben? Und sollten wir den Zutritt zu der Wohnung nach Bedürftigkeit regeln? Die Antwort lautet ganz offenkundig: Nein. Und zwar deswegen, weil wir uns gemeinsam wünschen, dass wir unter Normalbedingungen die Möglichkeit haben sollten, die eigene Wohnung, einschließlich des Zutritts und des Aufenthalts, zu kontrollieren.

Dieses individuelle moralische Eigentumsrecht verletzt nicht universelle Prinzipien der Gleichbehandlung. Diese sind mit legitimen Grenzen, hier den Grenzen meiner Wohnung, vereinbar. Individuelle (Eigentums-)Rechte dieser und anderer Art sind nicht absolut, wie Libertäre (vgl. Nozick 1974) meinen, sie können und müssen gegen andere moralische Gründe abgewogen werden. In unserem Fall könnte ich zum Beispiel eine moralische Pflicht zur Aufnahme des Obdachlosen dann haben, wenn es sich um eine klirrend kalte Winternacht handelte und zu befürchten wäre, dass der schon kränkelnde Obdachlose eine Nacht im Freien mit dem Leben bezahlen müsste. In Kriegs- und Nachkriegszeiten wurden die Vorrechte von Wohnungseigentümern eingeschränkt, etwa in Gestalt von Zwangsbelegungen für ausgebombte Familien oder Flüchtlinge nach Ende des 
Zweiten Weltkrieges. Die bloße Tatsache, dass es von Seiten des Staates eine allgemeine, durch gesetzliche Regelungen oder zumindest behördliche Anweisungen gestützte Praxis gibt, ist ethisch relevant. Eine solche allgemeine Praxis löst das Problem der ethischen Unterbestimmtheit (Wer hat in welchem Maße welche moralischen Verpflichtungen gegenüber den Flüchtlingen oder Ausgebombten?) und erfolgt nach - im günstigsten Fall - nachvollziehbaren und gerechten Kriterien (Größe der Wohnung, bisherige Belegungsdichte, Bedürftigkeit der Unterzubringenden etc.). Entscheidungen von dazu Befugten (z. B. im Rahmen staatlicher Gesetze oder staatlicher institutioneller Praxis) haben eine stärker legitimierende Wirkung als die individuelle Entscheidung einer Person, Grenzen (hier die Grenzen einer Wohnung) zu überschreiten.

Betrachten wir ein anderes Analogiebeispiel, das im Anschluss an Peter Singer (2004) gelegentlich angeführt wird, um für eine sehr weitgehende Aufnahmepflicht gegenüber Migranten zu argumentieren: Jemand kommt an einem Teich vorbei und sieht, dass eine Person zu ertrinken droht. Dann ist es offenkundig seine Pflicht, den Ertrinkenden zu retten, auch wenn dies bedeutet, dass seine Kleidung dabei nass wird. Die wohlhabenden Länder haben eine moralische Pflicht, ihre Grenzen jedenfalls so lange offenzuhalten, bis die Belastungen durch die Aufnahme unzumutbar groß werden. Peter Singer und zahlreiche philosophische Befürworter offener Grenzen ${ }^{4}$ fügen die empirische und wohl auch meist zutreffende Einschätzung hinzu, dass die Unzumutbarkeitsgrenze, also die Grenze, ab der die Belastungen der aufnehmenden Gesellschaft unerträglich werden, angesichts des unterdessen etablierten Wohlstandes in den Reichtumsregionen der Welt sehr hoch angesetzt werden kann. Auch eine Verdoppelung oder Verdreifachung der Wohnbevölkerung in einem überschaubaren Zeitraum sollte dann nicht als unzumutbar gelten. ${ }^{5}$

4 Vgl. Carens (2013) oder Andreas Cassee in seiner soeben als Buch erschienenen Dissertation, Cassee (2016). Cassee kritisiert kommunitaristische (Walzer) und nationalistische (Miller, Kymlicka) Thesen gegen „open borders“ unter Rückgriff auf libertäre und liberalistische Argumente; vgl. dazu Miller (2016) u. Kymlicka (1995). Eine Zusammenstellung von Beiträgen zu dieser Debatte findet sich in Cassee/Goppel (2012).

5 Hier gehen die Kalkulationen natürlich stark auseinander. Wenn man den Familiennachzug für Immigranten zulässt (schon aus Gründen der sozialen und der Geschlechter-Balance, aber auch aus Gründen der Humanität liegt dies nahe), müsste mittelfristig damit gerechnet werden, dass je nach Zusammensetzung der einwandernden Gruppe in den Folgejahren zwei bis vier weitere Personen für jeden Migranten nachziehen. Bei einer Einwanderung in dem monatlichen Umfang, wie er sich zwischen der Öffnungsentscheidung der deutschen Bundeskanzlerin und der Schließung der Balkanroute durch die Visegrád-Staaten zeigte, würde sich die in Deutschland lebende Bevölkerung in den nächsten zehn Jahren in etwa verdoppeln. Auch wenn dies eine massive Belastung der sozialen Sicherungssysteme und der wirtschaftlichen Leistungskraft pro Kopf bedeuten würde, ist anzu- 
Damit scheinen zwei Analogieargumente vorzuliegen, die zu gegensätzlichen ethischen Konsequenzen führen. Bevor wir versuchen, dieses Dilemma zu lösen, sei ausdrücklich angemerkt, dass ich solche Analogieargumente für zulässig halte. Der einfachste Weg wäre ja zu sagen, dass die unterschiedlichen Bereiche der Praxis jeweils von ganz unterschiedlichen Regeln geleitet sind und daher solche Analogien in die Irre führen. Ich bin deswegen der Auffassung, dass Analogieargumente dieser Art zulässig sind, weil die menschliche Praxis als Ganze kohärent zu sein hat. Man kann sich nicht lediglich darauf berufen, dass es sich um eine andere Praxis handelt, sondern man muss zusätzlich sagen, warum diese andere Praxis nach anderen ethischen Prinzipien geregelt werden sollte, um Analogieargumente abzuwehren.

Wenn jemand $\mathrm{zu}$ ertrinken droht, ist er mit dem Tode bedroht. Wenn der Obdachlose in einer klirrenden Winternacht mit dem Tode bedroht ist, wenn ich erwarten muss, dass er stirbt, wenn ich ihn aus der Wohnung weise, dann habe ich eine moralische Pflicht, ihn zu beherbergen. Selbst diese Pflicht scheint aber begrenzt zu sein. Wenn es sich zum Beispiel um einen schweren Alkoholiker handelt, der nur durch meine Fürsorge davon abgehalten werden kann, sich zu Tode zu trinken, kann daraus keine zeitlich unbegrenzte Pflicht zur Beherbergung abgeleitet werden. Selbst dann, wenn die Todesfolgen nicht eigenem Handeln (hier Alkoholgenuss), sondern den Umständen geschuldet sind, ist eine unbegrenzte Beherbergungspflicht nicht gerechtfertigt.

Es gibt ein berühmtes Argument für die Legitimität der Abtreibung, das folgende Analogie heranzieht: Eine Frau hat sich für eine Operation in eine Klinik begeben, und als sie aus der Narkose aufwacht, muss sie feststellen, dass ihr Blutkreislauf künstlich mit dem einer anderen Person (über Schläuche) verbunden wurde, und es stellt sich heraus, dass es sich dabei um einen weltberühmten Geiger handelt, der stürbe, wenn er nicht weiterhin von der Vitalität ihres HerzKreislauf-Systems profitieren könnte. Judith Jarvis Thomson (1971) hält es auch unter diesen Umständen für zulässig, dass die Frau entscheidet, nach Hause zu gehen und den Musiker sterben zu lassen. Das Recht auf Selbstbestimmung wird nicht einmal durch das Interesse einer erwachsenen, zudem der Menschheit nützlichen (,weltberühmter Geiger“) Person am Weiterleben aufgewogen. Ich, hier die schwanger gewordene Frau, kann mich gegen das werdende Leben in Gestalt einer Abtreibung entscheiden, unabhängig davon, welchen moralischen Status der Embryo hat. Selbst dann, wenn die Personeneigenschaft mit der Verschmelzung von Ei und Samenzelle etabliert wäre, selbst dann, wenn die Identität

nehmen, dass auch nach einer derart massiven Veränderung der Lebensstandard in Deutschland weit höher wäre als in den Ursprungsländern der Zugewanderten. 
der Person ab diesem Zeitpunkt feststünde, selbst dann, wenn der Embryo die gleiche Würde hätte wie ein geborenes menschliches Wesen oder - hier in der Geiger-Analogie - ein Erwachsener, hat das Selbstbestimmungsrecht ein größeres Gewicht als das Überlebensinteresse des Wesens, das von meinen Körperfunktionen abhängig ist.

Die Grenzen, die hier gezogen werden, sind andere als die der Wohnung, es sind Grenzen in Gestalt von Interventions-Verboten. Niemand hat das Recht, in dieser Weise in die persönliche Autonomie der Frau einzugreifen, selbst dann, wenn dieser Eingriff das Leben eines Menschen rettet. Man kann die vorrangige Rolle individueller Rechte in demokratischen Verfassungen als Grenzsetzungen dieser Art interpretieren: Was immer die Gründe sein mögen, die für eine Praxis sprechen - wenn sie individuelle Rechte einer Person verletzen, ist diese Praxis unzulässig. Natürlich kann sich die Trägerin individueller Rechte dazu entschließen, eine Intervention zu akzeptieren; in dem beschriebenen Fall kann sie sich entscheiden, für, sagen wir, neun Monate das Bett zu hüten und das Leben des bis dahin soweit Genesenen retten, der dann, ohne an das Herz-KreislaufSystem der Frau angedockt zu sein, überleben kann. Vom Geiger ist zu hoffen, dass er dann autonom leben kann; vom Neugeborenen ist das nicht zu erwarten. Es ist allerdings die Frau, die darüber entscheidet, weil sie das Recht hat, ein Leben nach eigenen Vorstellungen zu leben.

Die Analogie zur Migrationsthematik liegt auf der Hand: Es gehört zum kollektiven Selbstbestimmungsrecht einer Bürgerschaft, die sich in einem Staat organisiert hat, zu entscheiden, wie sie leben möchte, mit wem sie leben möchte, ob sie kulturelle, soziale und ökonomische Veränderungen akzeptiert oder nicht. ${ }^{6}$ Es gibt keine moralischen Gründe, die sie zwingen könnten, dieses Selbstbestimmungsrecht aufzugeben. Natürlich kann sie sich dafür entscheiden, die Veränderungen zu akzeptieren, die Grenzen zu öffnen, bislang nicht Beteiligte an der

\footnotetext{
6 Hier liegt der Einwand nahe, dass eine (Staats-)Bürgerschaft kein freiwilliger Zusammenschluss, sondern eine Zwangsgemeinschaft sei, die sich die allermeisten, die ihr angehören, nicht ausgesucht haben. Dies gilt aber nur hinsichtlich der Staatsangehörigkeit bei Geburt, die demokratische Staatsbürgerschaft als eine Form politischer Gemeinschaft und geteilter Praxis bezieht ihre Legitimation aus einem Konsens höherer Ordnung, einem geteilten Gerechtigkeitssinn. Dies ist jedenfalls der Kern der sogenannten vertragstheoretischen Rechtfertigung demokratischer Institutionen und Herrschaftsformen. Erst die faktische, implizite oder jedenfalls hypothetische Zustimmung aller Bürgerinnen und Bürger stiftet demokratische Legitimation. Diese (vertragstheoretische) Denkfigur aus dem 17. und 18. Jahrhundert (Hobbes, Locke, Rousseau, Kant) wurde 1971 von John Rawls mit seinem epochalen Werk A Theory of Justice erneuert, was weitere konkurrierende - vertragstheoretische Konzeptionen in der politischen Philosophie anregte, u. a. Buchanan (1974), Nozick (1974) und Gauthier (1986); vgl. dazu auch meine Überlegungen in NidaRümelin (1996).
} 
politischen Meinungsbildung teilhaben zu lassen, neue Kooperationsformen zu etablieren, Wohlfahrtsverluste hinzunehmen. Wenn ich den Ertrinkenden retten kann und mir dabei die Kleider nass mache, habe ich die Pflicht dazu. Wenn ich den Ertrinkenden nur retten kann, wenn ich meine individuelle Selbstbestimmung aufgebe, mein gesamtes Leben ändere, die Kontrolle über das verliere, was mir wertvoll ist, dann habe ich diese moralische Pflicht nicht mehr. Wenn wir das Elend der unteren beiden Milliarden der Weltbevölkerung durch offene Grenzen, durch Aufnahme diesseits des gerade noch Erträglichen bekämpften, wären Staat, Gesellschaft und Kultur, in denen wir leben, nicht mehr wiederzuerkennen. Man kann das wollen, aber man muss es nicht, es ist legitim, Grenzen zu setzen.

Dieses Argument für die Legitimität von Grenzen - unterschiedlichster Art ist, wohlgemerkt, nicht partikular oder kommunitaristisch. Hier wird nicht der Interessenstandpunkt eines Staates oder einer Gemeinschaft oder einer Person den Interessenstandpunkten anderer Staaten oder Gemeinschaften oder Personen vorgeordnet. Ich argumentiere nicht dafür, dass kollektive Loyalität nur möglich ist, wenn man das Eigene höher bewertet als das Fremde wie MacIntyre in „Ist Patriotismus eine Tugend?“ (MacIntyre 1993), ich argumentiere für ein universelles Recht auf individuelle und kollektive Selbstbestimmung, das allen (Individuen, Kollektiven, Staaten) gleichermaßen zukommt. Ohne Grenzen gibt es keine individuelle, kollektive, staatliche Selbstbestimmung und keine individuelle, kollektive oder staatliche Verantwortung, sondern die Strukturen der Verantwortungszuschreibung und der Akteure lösen sich auf. Ohne Grenzen werden die Lebensformen amorph, sie haben dann keine erkennbare Gestalt mehr, wir wissen dann nicht, wer welcher Akteur ist, wer wofür verantwortlich ist, welche Normen und Werte die jeweiligen Praktiken repräsentieren. Eine Gesellschaft aus perfekten Utilitaristen, die je für sich die Nutzensumme im Universum maximierten, bestünde nicht mehr aus Einzelpersonen, die Individuen wären nämlich ununterscheidbar, sie hätten keine eigenen Projekte, keine Normen und Werte, keine Identität. Es ist die - deontologische - Idee der individuellen Verantwortlichkeit, geschützt durch individuelle Rechte und Freiheiten und durch negative Pflichten der anderen, das heißt, durch moralische Gebote, die bestimmte Interventionen untersagen, die meine Autonomie gefährden könnten, die individuelle Autorschaft, die Fähigkeit, Autorin oder Autor des eigenen Lebens zu sein, sichert (vgl. Nida-Rümelin 1995 und Williams/Smart 1973).

$\mathrm{Zu}$ dieser Deontologie der Grenzen gehören die Abwehrrechte der Individuen gegen Interventionen nicht nur von Seiten des Staates, sondern auch von Seiten anderer Personen, und eben auch die konstitutiven Bedingungen kollektiver Autorschaft in Gestalt politischer Institutionen, Staaten, kultureller und anders verfasster Gemeinschaften. Ohne Struktur, ohne legitime und akzeptierte Grenzen keine Autorschaft, keine Zurechenbarkeit, keine Verantwortlichkeit, kein Respekt 
und keine Würde. Das so sympathische Plädoyer für Grenzenlosigkeit, die These, dass Grenzen grundsätzlich illegitim seien, weil sie Unterschiede aufrechterhielten (vgl. Moses 2006), lässt sich bei genauer Betrachtung ethisch nicht legitimieren.

Wie steht es aber um die Ungerechtigkeiten, die durch die Grenzen entstehen? Ist es nicht ungerecht, dass Menschen, die in einem bestimmten Land geboren sind, deswegen geringere Chancen auf Wohlergehen haben als andere? Verlangt nicht das Prinzip des Ausgleichs natürlicher oder zufälliger Unterschiede, um Chancengleichheit sichern zu können, dass Grenzen verschwinden?

Auch innerhalb einer nationalstaatlich verfassten Gesellschaft bestehen massive Ungleichheiten als Folge der Tatsache, dass man in eine bestimmte Familie hineingeboren wird, von einem bestimmten soziokulturellen Milieu umgeben ist, möglicherweise ungünstigen elterlichen Entscheidungen den eigenen Bildungsweg betreffend ausgesetzt war, etwas erbt oder nicht, genetisch begünstigt oder benachteiligt ist usw. Diese Unterschiede, in der englischsprachigen Philosophie der Gegenwart oft als natural luck bezeichnet, sollten in einer gerechten Gesellschaft so weit als möglich ausgeglichen werden: Den sozial Benachteiligten sollten besondere Mittel zur Verfügung stehen, um ihren Konkurrenznachteil ausgleichen zu können, Kinder mit Behinderungen sollten mehr staatliche Ressourcen binden dürfen, um sich gut entwickeln zu können, als Kinder ohne Behinderungen usw. Es gibt jedoch auch hier enge Grenzen des Zulässigen egalitaristischer Praxis. So wäre es ethisch unzulässig, Kinder auf die Familien umzuverteilen, Kinder mit genetischen Nachteilen in Familien mit sozioökonomischen Vorteilen zu verpflanzen und umgekehrt. Es wäre unzulässig, Heranwachsende mehrfach aus ihren Familienbezügen herauszunehmen und sie mit ungünstigeren beziehungsweise günstigeren Lebensbedingungen $\mathrm{zu}$ konfrontieren, um eine Gleichheit der Startbedingungen herzustellen. Diese und andere hyperegalitaristische Maßnahmen wären deswegen unzulässig, weil sie die wünschenswerten Strukturen einer humanen Gesellschaft, der Zusammengehörigkeit, der individuellen und kollektiven Selbstbestimmung zerstören würden. Eine humane Gesellschaft ist von der Balance zwischen Gleichheit und Differenz geprägt. Differenzen ergeben sich aus dem individuellen und kollektiven Recht auf Selbstbestimmung; sie vollkommen zu eliminieren, hieße, inhumane, individuelle und kollektive Verantwortlichkeit zerstörende Maßnahmen zu ergreifen.

Der Egalitarismus im Sinne des Gebots der Gleichbehandlung und der Gleichverteilung - es sei denn, es gäbe Gründe für eine Ungleichverteilung - muss mit den Differenzen der individuell und kollektiv gestalteten Lebensformen und ihren Zufälligkeiten vereinbar sein, sonst schlägt er in eine inhumane Praxis um. Wenn wir aber die Auflösung von Familienstrukturen, das Auseinanderreißen von Freund- 
schaften und Nachbarschaften im Dienste gleicher Chancen ablehnen, dann akzeptieren wir die ethische Relevanz von Strukturen, von Zugehörigkeiten und Abgrenzungen. Auch dann, wenn es der sozialen Emanzipation eines Individuums zuträglich wäre, wenn es zum Freundeskreis eines einflussreichen anderen Individuums gehörte, gibt es keine moralische Pflicht der Aufnahme in diesen Freundeskreis. Das Recht, seine Freunde selbst zu bestimmen, ist ein wesentliches Merkmal autonomer Lebensführung, es hat Vorrang gegenüber der Realisierung von Gleichheitspostulaten. Erst recht gilt dies für Eheschließungen. Über viele Jahrzehnte hinweg wurde soziale Mobilität teilweise dadurch gesichert, dass insbesondere Frauen über eine Eheschließung ihre sozioökonomische Situation verbesserten bzw. die soziale Schicht, der sie entstammten, hinter sich ließen. Dies ist heute weitgehend zum Erliegen gekommen. Soziologen sprechen von Endogamie, im Sinne einer Einheirat in dieselbe soziale Schicht, was vermutlich damit zusammenhängt, dass die Abstiegsängste größer geworden sind und die Absicherung gegen diesen Abstieg durch vergleichbare ökonomische Leistungskraft zumindest unbewusst eine Rolle bei Eheschließungen spielt. Aber auch dann, wenn die Heirat über unterschiedliche sozioökonomische Milieus hinweg ein wichtiger Beitrag für eine egalitärere und sozial mobilere Gesellschaft wäre, könnte niemand darauf verpflichtet werden. Das individuelle und hier auch das kollektive (ein Kollektiv, das zwei Personen umfasst) Selbstbestimmungsrecht hat Vorrang.

Wir sollten uns das Zusammenleben der menschlichen Gesellschaft diesseits und jenseits nationaler Grenzen und über die Zeiten der Menschheitsgeschichte hinweg wie eine Ansammlung unterschiedlicher Dichte von Myriaden von Teilchen in einer bewegten Flüssigkeit vorstellen. An manchen Stellen formen sich diese Teilchen zu dichten Klumpen, an anderen dünnen sie aus, manche bleiben über längere Zeiträume hinweg in enger räumlicher Nähe, andere entfernen sich voneinander und begegnen sich nie wieder, andere sind hoch mobil und nähern sich der einen oder anderen Verklumpung nur vorübergehend an, um dann zu einer anderen zu wechseln. Die Ströme der Flüssigkeit werden von Gefäßen strukturiert, zwischen denen es mehr oder weniger starken Austausch gibt. Die Bewegungs- und Interaktionsmuster ändern sich nicht nur von Gefäß zu Gefäß, sondern auch zwischen den unterschiedlichen Verklumpungen.

Der Vorzug dieser Metapher ist, dass sie das Graduelle, die permanent fließenden Übergänge, das Wechselverhältnis von Struktur (der Gefäße) und Strömungsbild vor Augen führt. Den jeweiligen Verklumpungen entsprechen soziale Nahbereiche und kulturelle Gemeinschaften verschiedenster Art. Dem Wechsel der Teilchen von der einen zur anderen Verklumpung und der Überlagerung unterschiedlicher Verklumpungen im Strömungsgeschehen entsprechen die Kreolisierungsprozesse und die Kosmopolitisierung der Weltgesellschaft (vgl. Beck 2004 und Beck/Grande 2004). Die einzelnen Gefäße sind nicht hermetisch 
voneinander abgeschlossen, sie sind miteinander verbunden, aber in unterschiedlichem Ausmaß. Nur sehr wenige Staaten der Welt können sich gegenüber der globalen Migration abschließen.

Die einzelnen Verklumpungen sind durch spezifische Formen von Interaktion geprägt, die an den Rändern ausdünnen und oft in fließendem Übergang zu anderen Strukturen der Interaktion und der damit einhergehenden normativen Stellungnahmen stehen. Es lassen sich jedoch auch gemeinsame Muster über alle Verklumpungs- und Gefäßstrukturen hinweg erkennen. Man mag dies als das Bild einer globalen Zivilgesellschaft interpretieren. Diese entsteht nicht durch einen besonderen Status, sondern in Fortsetzung bestehender Bindungen und Interaktionen, die im globalen Maßstab ephemerer werden und eine Normativität voraussetzen, die partikulare Bindungen überwölbt.

Wir haben in diesem Bild Gefäßstrukturen, Interaktionsstrukturen, institutionell verfestigte kollektive Identitäten, etwa in Gestalt einer verbindlichen und sanktionierten Rechtsordnung über alle kulturellen und regionalen Gemeinschaften hinweg, und eine weiche Strukturbildung, meist ohne oder nur mit einer schwach ausgeprägten institutionellen Verfestigung: das, was üblicherweise als kulturelle Identität bezeichnet wird. Diese beiden strukturbildenden Merkmale sind nicht unabhängig voneinander, aber man darf sie nicht identifizieren. Das ethnizistische Missverständnis des Nationalstaates identifiziert diesen mit einer partikularen, kulturell bestimmten Lebensform und empfindet daher jede Vervielfältigung kultureller und regionaler Gemeinschaften als eine Bedrohung. Diese ethnizistische Fassung des Nationalstaates tendiert zu einer Planierung und Nivellierung, im Konfliktfalle zur Unterdrückung partikularer (genauer: partikularerer) Gemeinschaften, und provoziert dadurch wiederum Revolten, die sich über ethnische Identität legitimieren. Dieses Muster prägt den Kurdenkonflikt in der Türkei ebenso wie die separatistischen Bewegungen etwa der Katalanen oder der Schotten in Europa. Das, was durch Nivellierung und Assimilation zum Verschwinden gebracht werden soll, formiert sich als seinerseits „national“ interpretierter Widerstand neu. Statt als „Bergtürken“ die Differenzen der Lebensform langsam abklingen zu lassen, wie es der türkische Nationalismus erwartete und forderte, formiert sich eine virtuelle kurdische Nation, die nicht nur die Türkei, sondern drei weitere Staaten um ihre territoriale Integrität fürchten lässt.

Auch der Umgang mit religiösen Gemeinschaften folgt demselben Muster: Solche Gemeinschaften, die sich marginalisiert fühlen, die ihre eigenen Gotteshäuser nicht errichten dürfen, die sich ins Private zurückziehen müssen, die sich gewissermaßen den Blicken der Mehrheitskultur entziehen müssen, um ihren religiösen Bräuchen und spirituellen Praktiken nachzugehen, sind vor die Alternative gestellt, sich zu assimilieren und einen Teil ihrer Identität aufzugeben oder gerade das Verdrängte zum Zentrum einer wie auch immer imaginierten oppo- 
sitionellen Identität zu machen. In dieser Hinsicht scheint mir die amerikanische Praxis in einer im hohen Maße von religiösen Empfindungen und Praktiken geprägten Gesellschaft geradezu vorbildlich zu sein: Gotteshäuser unterschiedlichster Provenienz, nicht nur christliche, stoßen - bislang - nicht auf Widerstand, und die Vielfalt christlicher Konfessionen lässt die christliche Mehrheitsgesinnung für andere Religionsgemeinschaften nicht als bedrohlich erscheinen.

In den nichtmonotheistischen Religionen ist die Kombination unterschiedlicher religiöser Traditionen, ja, die multireligiöse Nutzung derselben Tempelanlagen ausgeprägter. Shintoistische und buddhistische, auch taoistische und konfuzianische Glaubensinhalte und -riten werden nicht als unvereinbar empfunden. Die Missionierung der Angehörigen anderer Glaubensrichtungen ist in diesen Kulturregionen unüblich. Auch die mit der religiösen Identität verbundene kulturelle Abschließung ist in den monotheistischen Religionsgemeinschaften verbreiteter und reicht bis zur massiven Sanktionierung, die sich in einigen Rechtsordnungen islamischer Staaten als Todesstrafe für die Abkehr vom muslimischen Glauben gehalten hat (Apostasie). Aber auch die Praxis der Endogamie, also die Praxis der Verheiratung innerhalb einer Religionsgemeinschaft, ist eine historische Gemeinsamkeit der drei monotheistischen Wüstenreligionen. ${ }^{7}$

Die gesellschaftliche Konvivialität unterschiedlicher Religionsgemeinschaften setzt bei allen Differenzen der Lebensformen und der Bewertungen einen Konsensus höherer Ordnung voraus, der sich auf den Umgang mit Religion und kulturellen Differenzen bezieht. Erst dieser Konsensus höherer Ordnung stiftet Zivilität, einen zivilen Umgang über Wertungsdifferenzen hinweg. Aber auch diese zivile Praxis angesichts religiöser Differenzen muss in der Alltagskultur verankert sein. Eine religiös motivierte Apartheid mit separaten Orten der Begegnung, des Amüsements, der Freizeit und der Arbeit wäre mit dieser Form der Zivilität unvereinbar. Auch die Separierung in religiös einheitliche Viertel in den Großstädten mag manche Konflikte erst gar nicht entstehen lassen, ist aber ein Hindernis für eine gemeinsame, bürgerschaftliche (politisch verfasste) Identität.

Die Hoffnung liberaler Philosophen, dass sich das Politische und das Kulturelle sorgsam trennen lassen, trügt. Wir sind mit einem Kontinuum kleiner Partikularitäten vom Nahbereich über kulturelle und religiöse Gemeinschaften bis zu den normativen Konstitutiva ziviler Staatlichkeit und globaler Bürgerschaft konfrontiert. Die Praxis politischer Partizipation, also eine republikanisch verfasste Demokratie, kann in der Tat zur Befriedung kultureller und religiöser Konflikte

7 Diese Bezeichnung spielt auf die gleiche Ursprungsregion an, die ihre Gemeinsamkeiten, aber auch Unverträglichkeiten erklärt. 
einen wesentlichen Beitrag leisten; sie über den Nationalstaat auszudehnen, ist ein - kosmopolitisches - Postulat.

\section{Bibliographie}

Beck, Ulrich (2000): „The Cosmopolitan Perspective: Sociology of the Second Age of Modernity“. In: British Journal of Sociology 51, S. 78-105.

Beck, Ulrich (2004): Der kosmopolitische Blick oder: Krieg ist Frieden. Frankfurt am Main: Suhrkamp.

Beck, Ulrich/Grande, Edgar (2004): Das kosmopolitische Europa. Frankfurt am Main: Suhrkamp.

Brock, Gillian (2009): Global Justice. A Cosmopolitan Account. Oxford: Oxford University Press.

Buchanan, James (1974): The Limits of Liberty. Between Anarchy and Leviathan. Chicago: University of Chicago Press.

Carens, Joseph (2013): The Ethics of Immigration. Oxford: Oxford University Press.

Cassee, Andreas (2016): Globale Bewegungsfreiheit. Ein philosophisches Plädoyer für offene Grenzen. Berlin: Suhrkamp.

Cassee, Andreas/Goppel, Anna (Hrsg.) (2012): Migration und Ethik. Münster: mentis.

Gauthier, David (1986): Morals by Agreement. Oxford: Oxford University Press.

Kant, Immanuel (1969): „Zum Ewigen Frieden“ [1. Aufl. 1795]. In: Kants Werke. Bd. 8. Hrsg. von der Preußischen Akademie der Wissenschaften. Berlin, New York: De Gruyter, S. 341-386.

Kymlicka, Will (1995): Multicultural Citizenship. A Liberal Theory of Minority Rights. Oxford: Oxford University Press.

MacIntyre, Arthur (1993): „Ist Patriotismus eine Tugend?“. In: Axel Honneth (Hrsg.): Kommunitarismus. Eine Debatte über die moralischen Grundlagen moderner Gesellschaften. Frankfurt am Main: Suhrkamp, S. 84-102.

Miller, David (2000): Citizenship and National Identity. Oxford: Oxford University Press. Miller, David (2016): Strangers in Our Midst. The Political Philosophy of Immigration. Cambridge, MA: Harvard University Press.

Moses, Jonathon W. (2006): International Migration. Globalizations' Last Frontier. London: Zed Books.

Nida-Rümelin, Julian (1995): Kritik des Konsequentialismus. München: R. Oldenbourg. Nida-Rümelin, Julian (1999): Demokratie als Kooperation. Frankfurt am Main: Suhrkamp. Nida-Rümelin, Julian (2006): „Zur Philosophie des Kosmopolitismus“. In: Zeitschrift für internationale Beziehungen 13, S. 231-238.

Nozick, Robert (1974): Anarchy. State. Utopia. New York: Basic Books.

Rawls, John (1971): A Theory of Justice. Cambridge, MA: Harvard University Press.

Singer, Peter (2004): One World. The Ethics of Globalization. New Haven: Yale University Press. Thomson, Judith J. (1971): „A Defense of Abortion“. In: Philosophy \& Public Affairs 1. Nr. 1, S. $47-66$.

Williams, Bernard/Smart, J.J.C. (1973): Utilitarianism. For and Against. Cambridge: Cambridge University Press. 